Elect. Comm. in Probab. 5 (2000) 51-55

ELECTRONIC

COMMUNICATIONS in PROBABILITY

\title{
THE CONVEX MINORANT OF THE CAUCHY PROCESS
}

\author{
JEAN BERTOIN \\ Laboratoire de Probabilités et Modèles Aléatoires, Université Pierre et Marie Curie, 4, Place \\ Jussieu, F-75252 Paris Cedex 05, France, \\ email: jbe@ccr.jussieu.fr
}

submitted Nov. 11, 1999. Final version accepted Jan. 20, 2000.

AMS 1991 Subject classification: 60J30

Cauchy process, convex minorant

\section{Abstract}

We determine the law of the convex minorant $\left(M_{s}, s \in[0,1]\right)$ of a real-valued Cauchy process on the unit time interval, in terms of the gamma process. In particular, this enables us to deduce that the paths of $M$ have a continuous derivative, and that the support of the Stieltjes measure $d M^{\prime}$ has logarithmic dimension one.

For a given real-valued function $f$ defined on some interval $I \subseteq \mathbb{R}$, one calls the convex minorant of $f$ the largest convex function on $I$ which is bounded from above by $f$. The case when $I=[0, \infty[$ and $f$ is a sample path of Brownian motion has been studied in depth by Groeneboom [11], see also Pitman [13] and Çinlar [6]. In particular, it has been shown in these works that the convex minorant of Brownian motion is almost surely a piecewise linear function on the open interval ]0, $\infty$, and that the distribution of its derivative can be characterized in terms of a certain process with independent (non-stationary) increments. The more general case when $f$ is a sample path of a Markov process (respectively, a Lévy process) has been considered by Bass [1] (respectively, by Nagasawa and Tanaka [12]).

In this note, we carry out a similar study for the Cauchy process on the unit time interval; we shall establish in particular a simple connection with the gamma process which yields several interesting consequences. More precisely, we shall see that the derivative of the convex minorant of the Cauchy process is continuous on ]0, 1 [, specify its behavior near the boundary points 0 and 1 , and determine the exact Hausdorff measure of the set of points on which it increases.

Let $\left(C_{s}, s \in[0,1]\right)$ be a standard one-dimensional Cauchy process and $\left(M_{s}, s \in[0,1]\right)$ denote its convex minorant. The right-derivative $\left(M_{s}^{\prime}, s \in[0,1[)\right.$ is an increasing process with rightcontinuous paths, and we write

$$
\mu_{x}=\inf \left\{s \in \left[0,1\left[: M_{s}^{\prime}>x\right\}, \quad x \in \mathbb{R}\right.\right.
$$


for its right-continuous inverse. Recall that the standard gamma process, $\left(\gamma_{s}, s \in[0,1]\right)$ is a subordinator with marginal distributions

$$
\mathbb{P}\left(\gamma_{s} \in d x\right)=\Gamma(s)^{-1} x^{s-1} \mathrm{e}^{-x} d x, \quad x \geq 0 .
$$

Our analysis relies on the following.

Lemma 1 The process $\left(\mu_{x}, x \in \mathbb{R}\right)$ has the same law as $\left(\gamma_{\rho(x)} / \gamma_{1}, x \in \mathbb{R}\right)$ where $\rho(x)=\frac{1}{2}+$ $\frac{1}{\pi} \arctan x$.

Proof: It is convenient for our purpose to first work with a Cauchy process defined on the time interval $\left[0, \infty\left[\right.\right.$, and to denote its natural filtration by $\left(\mathcal{F}_{s}\right)_{s \geq 0}$. For every $x \in \mathbb{R}$ and $s \geq 0$, write $C_{s}^{(x)}=C_{s}-x s$ for the Cauchy process with constant drift $-x$, and set $I_{s}^{(x)}=\inf _{0 \leq r \leq s} C_{s}^{(x)}$ for its infimum process. Introduce the so-called ladder-time set

$$
\mathcal{L}^{(x)}=\left\{s \geq 0: I_{s}^{(x)}=C_{s}^{(x)}\right\}
$$

On the one hand, $\mu_{x}$ is the (a.s. unique) instant in $[0,1]$ at which the Cauchy process with drift $C^{(x)}$ reaches its overall infimum on $[0,1]$, so

$$
\mu_{x}=\sup \left\{s \in[0,1]: s \in \mathcal{L}^{(x)}\right\}
$$

On the other hand, it is immediately seen that $\left(\mathcal{L}^{(x)}, x \in \mathbb{R}\right)$ is a nested family of random sets, in the sense that $\mathcal{L}^{(x)} \subseteq \mathcal{L}^{\left(x^{\prime}\right)}$ for $x \leq x^{\prime}$. Moreover, the strong Markov property of the Cauchy process easily entails the following regenerative property. Consider an arbitrary finite sequence $x_{1} \leq x_{2} \leq \ldots \leq x_{n}$ of increasing real numbers, and $T$ a stopping time in the filtration $\left(\mathcal{F}_{s}\right)_{s \geq 0}$ such that $T \in \mathcal{L}^{\left(x_{1}\right)}$ a.s. Then the shifted ladder time sets

$$
\mathcal{L}^{\left(x_{i}\right)} \circ \theta_{T}:=\left\{s \geq 0: s+T \in \mathcal{L}^{\left(x_{i}\right)}\right\}, \quad i=1, \ldots, n
$$

are jointly independent of $\mathcal{F}_{T}$ and have the same (joint) distribution as $\mathcal{L}^{\left(x_{1}\right)}, \ldots, \mathcal{L}^{\left(x_{n}\right)}$. Note also that the probability $\mathbb{P}\left(C_{s}^{\left(x_{i}\right)} \leq 0\right)$ is the same for all $s>0$, and if we denote this quantity by $\rho\left(x_{i}\right)$, then

$$
\rho\left(x_{i}\right)=\int_{-\infty}^{x_{i}} \frac{d x}{\pi\left(1+x^{2}\right)}=\frac{1}{2}+\frac{1}{\pi} \arctan x_{i} .
$$

Hence each regenerative set $\mathcal{L}^{\left(x_{i}\right)}$ is stable with index $\rho\left(x_{i}\right)$, i.e. it can be identified with the closed range of some stable subordinator with index $\rho\left(x_{i}\right)$, see Lemma VIII.1 in [2].

We have checked that the general framework of [3] applies to the present setting, and Proposition 9 there entails that the law of the $n$-tuple $\left(\mu_{x_{2}}-\mu_{x_{1}}, \ldots, \mu_{x_{n}}-\mu_{x_{n-1}}, 1-\mu_{x_{n}}\right)$ is the $n$-dimensional Dirichlet distribution with parameters $\left(\rho\left(x_{2}\right)-\rho\left(x_{1}\right), \ldots, \rho\left(x_{n}\right)-\rho\left(x_{n-1}\right), 1-\right.$ $\left.\rho\left(x_{n}\right), \rho\left(x_{1}\right)\right)$, that is the same as that of

$$
\frac{1}{\gamma_{1}}\left(\gamma_{\rho\left(x_{2}\right)}-\gamma_{\rho\left(x_{1}\right)}, \ldots, \gamma_{\rho\left(x_{n}\right)}-\gamma_{\rho\left(x_{n-1}\right)}, \gamma_{1}-\gamma_{\rho\left(x_{n}\right)}\right)
$$

It follows that the two processes in the statement have the same finite-dimensional distributions, and as both are increasing and right-continuous, they have the same law. 
Remark. Two different nested families of stable regenerative sets have been considered in [5]. In this direction, we point out that the argument in section 4 there can be adapted to give an alternative proof of Lemma 1.

Our main result derives immediately from Lemma 1 and the fact that the gamma process has strictly increasing paths with probability one.

Theorem 2 The process $\left(M_{s}^{\prime}, 0<s<1\right)$ is continuous and has the same law as

$$
-\cot \left(\pi L\left(s \gamma_{1}\right)\right), \quad 0<s<1
$$

where $\gamma$ is a standard gamma process and

$$
L(x)=\inf \left\{s \geq 0: \gamma_{s}>x\right\}, \quad x \geq 0
$$

denotes its inverse process.

Proof: It follows from Lemma 1 that the process $\left(\mu_{x}, x \in \mathbb{R}\right)$ has strictly increasing paths with probability one; as a consequence, $\left(M_{s}^{\prime}, 0<s<1\right)$ can be recovered from $\left(\mu_{x}, x \in \mathbb{R}\right)$ by the identity

$$
M_{s}^{\prime}=\inf \left\{x \in \mathbb{R}: \mu_{x}>s\right\} .
$$

This entails the continuity of $M^{\prime}$ and the stated identity in law (again by Lemma 1).

As a check, let us calculate the distribution of the variable $C_{1}$, which coincides with $M_{1}=$ $\int_{0}^{1} M_{s}^{\prime} d s$. It follows from Theorem 2 that the latter has the same law as

$$
\tilde{M}_{1}:=-\frac{1}{\gamma_{1}} \int_{0}^{1} \cot (\pi s) d \gamma_{s} .
$$

Next, recall that $\gamma_{1}$ has an exponential distribution with parameter 1 and is independent of the Dirichlet process $\left(\gamma_{s} / \gamma_{1}, s \in[0,1]\right)$, and a fortiori of $\tilde{M}_{1}$. It follows that for every $\lambda \in \mathbb{R}$, the Stieltjes transform (i.e. the iterated Laplace transform) of $M_{1}$ is given by

$$
\mathbb{E}\left(1 /\left(1+i \lambda M_{1}\right)\right)=\mathbb{E}\left(\exp \left\{-i \lambda \gamma_{1} \tilde{M}_{1}\right\}\right)=\mathbb{E}\left(\exp \left\{i \lambda \int_{0}^{1} \cot (\pi s) d \gamma_{s}\right\}\right) .
$$

Using the identity $\mathbb{E}\left(\exp \left\{i \lambda \gamma_{s}\right\}\right)=(1-i \lambda)^{-s}$, we see that the right-hand side equals

$$
\exp \left\{-\int_{0}^{1} \log (1-\mathbf{i} \lambda \cot (\pi s)) d s\right\}=\exp \left\{-\int_{-\infty}^{\infty} \log (1+\mathbf{i} \lambda x) \frac{d x}{\pi\left(1+x^{2}\right)}\right\} .
$$

When $\lambda>0$ (respectively, $\lambda<0$ ), the function $z \rightarrow\left(1+z^{2}\right)^{-1} \log (1+i \lambda z)$ is meromorphic on the lower (respectively, upper) complex half-plane with a single pole at $z=-\mathrm{i}$ (respectively, at $z=\mathrm{i}$ ). Using a contour integral, one gets

$$
\int_{-\infty}^{\infty} \log (1+\mathrm{i} \lambda x) \frac{d x}{\pi\left(1+x^{2}\right)}=\log (1+|\lambda|) .
$$

Putting the pieces together, we conclude that

$$
\mathbb{E}\left(1 /\left(1+i \lambda M_{1}\right)\right)=1 /(1+|\lambda|),
$$


and hence that $M_{1}$ follows the standard Cauchy distribution.

The same method applies for instance to calculate the distribution of the length

$$
\ell=\int_{0}^{1} \sqrt{1+\left(M_{s}^{\prime}\right)^{2}} d s=\int_{-\infty}^{\infty} \sqrt{1+x^{2}} d \mu_{x}
$$

of the graph of the convex minorant. One gets after a few lines of elementary calculation

$$
\mathbb{E}(1 /(1+q \ell))=\exp \left\{-\int_{0}^{1} \log (1+q / \sin (\pi \theta)) d \theta\right\} .
$$

Next, we deduce from known properties about the regularity of $L$ the following results on the rate of growth of $M^{\prime}$ near the boundary points 0 and 1.

Corollary 3 With probability one, we have

$$
\liminf _{s \rightarrow 0+} \frac{\left|M_{s}^{\prime}\right| \log \log \log 1 / s}{\log 1 / s}=\liminf _{s \rightarrow 0+} \frac{M_{1-s}^{\prime} \log \log \log 1 / s}{\log 1 / s}=\frac{1}{\pi} .
$$

Moreover, if $f:[0, \infty[\rightarrow[0, \infty[$ is an increasing function such that $t \rightarrow f(t) / t$ decreases, then both

$$
\limsup _{s \rightarrow 0+}\left|M_{s}^{\prime}\right| f(s) \quad \text { and } \quad \limsup _{s \rightarrow 0+}\left|M_{1-s}^{\prime}\right| f(s)
$$

equal 0 or $\infty$ according as the integral $\int_{0+} f(x) x^{-1} d x$ converges or diverges.

Proof: The lim inf result follows from Theorem 2 , the estimate $\cot (\pi x) \sim 1 /(\pi x)$ as $x \rightarrow 0+$ and the law of the iterated logarithm for $L$,

$$
\limsup _{s \rightarrow 0+} \frac{L(s) \log 1 / s}{\log \log \log 1 / s}=1
$$

(the latter can be seen for instance from Theorem 1 in [4] and the fact that the Laplace exponent of the gamma process is $\log (1+\cdot))$. The proof of the lim sup result is similar, using Theorem 2 on page 321 in [10].

One can also complete Corollary 3 by further studying the regularity of $M^{\prime}$ on ]0,1[. More precisely, it is easily seen that for each fixed $s \in] 0,1\left[, M^{\prime}\right.$ remains constant on some neighborhood of $s$ a.s. So to have a non-trivial result, we focus on points at which $M^{\prime}$ increases, that is of the type $s=\mu_{x}$ for some given $x \in \mathbb{R}$. Then a variation of the argument for the proof of Corollary 3 yields

$$
\limsup _{s \rightarrow 0+} \frac{\left(M_{s+\mu_{x}}^{\prime}-x\right) \log 1 / s}{\log \log \log 1 / s}=\pi\left(1+x^{2}\right) \quad \text { a.s. }
$$

Finally, we turn our interest to the support of the Stieltjes measure $d M^{\prime}$, that is to the set of points at which the slope of the convex minorant increases.

Corollary 4 Introduce the function

$$
h(s)=\frac{\log \log \log 1 / s}{\log 1 / s}
$$

Then with probability one, for every $x \in] 0,1\left[\cap \operatorname{Supp}\left(d M^{\prime}\right)\right.$ and $\left.\varepsilon \in\right] 0, x \wedge(1-x)[$, the h-Hausdorff measure of $[x-\varepsilon, x+\varepsilon] \cap \operatorname{Supp}\left(d M^{\prime}\right)$ is positive and finite. 
In particular, $\operatorname{Supp}\left(d M^{\prime}\right)$ is a random closed set with logarithmic Hausdorff dimension 1.

Proof: It follows from Theorem 2 that $\operatorname{Supp}\left(d M^{\prime}\right)=\left\{\mu_{x}, x \in \mathbb{R}\right\}^{\text {cl }}$ is distributed as the normalized closed range of a gamma process on the unit time interval. It is a consequence of a general result by Fristedt and Pruitt [9] that there is some constant $c>0$ such that for every $t>0$ the $h$-Hausdorff measure of $\left\{\gamma_{s}, 0 \leq s \leq t\right\}^{\text {cl }}$ is $c t$. This entails our claim.

Remark. Theorem 2 and Corollaries 3 and 4 bear the same flavor as the results of Cranston et al. [7] and Evans [8] on the convex hull of planar Brownian path on the unit time interval (recall e.g. that the convex hull is a $\mathcal{C}^{1}$ curve and that the set of times in $[0,1]$ at which the Brownian path touches the convex hull has Hausdorff dimension zero). These similarities are certainly not surprising, considering the close connections between the planar Brownian motion and the linear Cauchy process.

\section{References}

[1] R. Bass: Markov processes and convex minorants. Séminaire de Probabilités XVIII (1984), 28-41.

[2] J. Bertoin: Lévy Processes. Cambridge University Press, 1996.

[3] J. Bertoin: Renewal theory for embedded regenerative sets. To appear in Ann. Probab. (1999)

[4] J. Bertoin and M. E. Caballero: On the rate of growth of subordinators with slowly varying Laplace exponent. Séminaire de Probabilités XXIX (1995), 125-132.

[5] J. Bertoin and J. Pitman: Two coalescents derived from the ranges of stable subordinators. To appear in Electronic Journal of Probability.

[6] E. Çinlar: Sunset over Brownistan. Stochastic Process. Appl. 40 (1992), 45-53.

[7] M. Cranston, P. Hsu and P. March: Smoothness of the convex hull of planar Brownian motion. Ann. Probab. 17 (1989) 144-150.

[8] S. N. Evans: On the Hausdorff dimension of Brownian cone points. Math. Proc. Camb. Philos. Soc. 98 (1985), 343-353.

[9] B. E. Fristedt and W. E. Pruitt: Lower functions for increasing random walks and subordinators. Z. Wahrscheinlichkeitstheorie verw. Gebiete 18 (1971), 167-182.

[10] I. I. Gihman and A. V. Skorohod (1975). The theory of stochastic processes II. Springer, Berlin.

[11] P. Groeneboom: The concave majorant of Brownian motion. Ann. Probab. 11 (1983), 1016-1027.

[12] M. Nagasawa and H. Tanaka: Concave majorants of Lévy processes. Preprint (1999).

[13] J. W. Pitman: Remarks on the convex minorant of Brownian motion. In: Seminar on stochastic processes (Evanston, 1982), pp. 219-227, Progr. Probab. Statist., 5, Birkhäuser Boston, Boston, Mass., 1983. 